Revue d'histoire du XIXe siècle

Société d'histoire de la révolution de 1848 et des

révolutions du XIXe siècle

$53 \mid 2016$

Mobilités, savoir-faire et innovations

\title{
Exil, finances internationales et construction de l' Etat : les libéraux et « joséphins » espagnols (1813-1851)
}

Exile, International Finance and Statebuilding : the Spanish 'Joséphins' and Liberals (1813-1851)

Exil, internationales Finanzwesen und Staatsbildung: Die Liberalen und die spanischen «Josephinen » 1813-1851

Juan Pan-Montojo et Juan Luis Simal

\section{OpenEdition}

\section{Journals}

Édition électronique

URL : http://journals.openedition.org/rh19/5089

DOI : $10.4000 /$ rh 19.5089

ISSN : 1777-5329

Éditeur

La Société de 1848

Édition imprimée

Date de publication : 1 décembre 2016

Pagination : 59-77

ISSN : 1265-1354

Référence électronique

Juan Pan-Montojo et Juan Luis Simal, « Exil, finances internationales et construction de l' Etat : les libéraux et « joséphins » espagnols (1813-1851) », Revue d'histoire du XIXe siècle [En ligne], 53 | 2016, mis en ligne le 01 décembre 2018, consulté le 04 janvier 2020. URL : http://journals.openedition.org/ rh19/5089 ; DOI : 10.4000/rh19.5089 


\title{
JUAN PAN-MONTOJO ET JUAN LUIS SIMAL
}

\author{
Exil, finances internationales et construction \\ de l'Etat: les libéraux et «joséphins» espagnols \\ (1813-1851)
}

Les caractéristiques politiques et discursives de l'internationalisme libéral du XIX ${ }^{e}$ siècle ont été décrites par plusieurs historiens qui ont montré combien l'exil et la mobilité constituent des facteurs clés pour leur juste compréhension ${ }^{2}$. Toutefois, les dimensions économiques et, plus concrètement, les dimensions financières de l'émigration politique, n'ont pas fait l'objet d'une étude approfondie. Cet article propose une analyse financière de l'exil espagnol durant les restaurations absolutistes du règne de Ferdinand VII (1813-1833) et de ses conséquences ultérieures en termes politiques et institutionnels. On ne fera pas seulement référence par là aux activités économiques réalisées par les exilés, mais aussi aux interactions qui ont existé entre leurs projets politiques et leurs efforts pour trouver des financements, ainsi qu'aux trâces que leurs activités financières ont laissées dans différents domaines de la monarchie espagnole. Aussi s'intéressera-t-on à la façon dont les projets économiques des exilés se sont combinés avec leurs discours, leurs programmes et leur activisme politique, et à la manière dont ceci a affecté le processus de construction de l'État en Espagne.

Les exilés comptaient dans leurs rangs aussi bien ceux qui commençaient à être connus sous le nom de «libéraux», que ceux qui, en 1813 et dans les années qui ont suivi, avaient dû abandonner l'Espagne en raison de leur adhésion au parti du roi Joseph ${ }^{\text {er }}$ Bonaparte : les "joséphins" ou afrancesados. ${ }^{3}$ L'évolution politique de ce groupe a été, au cours des décennies postérieures, hétérogène - et une partie importante de ses membres n'a jamais

1. Cet article passe en revue quelques-uns des postulats et hypothèses initiales du projet de recherche "Deudex" porté par Juan Pan-Montojo à l'Universidad Autónoma de Madrid, "La dette extérieure comme mécanisme de transformation politique, sociale et culturelle : le monde ibérique et la Méditerranée orientale, 1814-1914» (HAR2015-66695-P).

2. Maurizio Isabella, Risorgimento in exile. Italian Emigrés and the Liberal International in the Post-Napoleonic Era, Oxford, Oxford University Press, 2009; Juan Luis Simal, Emigrados. España y el exilio internacional, 1814-1834, Madrid, Centro de Estudios Políticos y Constitucionales, 2012.

3. Nous préférons le terme "joséphin" dans le sens de partisan ou, tout du moins, de collaborateur politique du roi Joseph Ier Bonaparte. Dans le cadre d'un article où l'on va insister sur l'influence du modèle français, ce terme de "joséphin " nous semble plus approprié que celui, commun et péjoratif, d'afrancesado. 
soutenu de positions libérales, quelle que soit la définition que l'on donne de ce terme - et leurs relations avec leurs anciens ennemis de la période 1808-1813, les libéraux "patriotes», ont souvent été difficiles. Néanmoins, les "joséphins" ont joué, comme nous le verrons, un rôle important aussi bien dans la politique espagnole en France entre 1813 et 1833, que dans les changements politiques qui se sont produits en Espagne, en particulier à partir du Triennat libéral (1820-1823).

En prenant en considération ces différents acteurs, il s'agira d'analyser le lien entre politique et finances en montrant qu'il a constitué un aspect décisif de l'exil. L'exil peut se définir comme une expérience à travers laquelle se sont articulés des réseaux et redéfinis des projets qui, comme nous le défendons et tenterons de le prouver, ont eu des conséquences importantes pour la constitution de l'État libéral et la création d'un marché national en Espagne, à un moment où État et marché connaissaient une adaptation importante à l'environnement post-révolutionnaire. Nous croyons que cet examen est fondamental pour comprendre les intérêts du libéralisme en exil ainsi que pour saisir les projets de construction étatique et nationale portés par les exilés. Par ailleurs, comme cette étude fait partie intégrante de l'histoire des finances internationales, nous prêterons également attention aux traits transnationaux du libéralisme économique et du secteur bancaire.

Plus précisément, l'article débute par l'examen des activités financières réalisées par les exilés libéraux espagnols durant la décennie 1823-1833, période durant laquelle l'appel à des capitaux internationaux pour financer des projets politiques a été soumis à une concurrence beaucoup plus marquée. Pour obtenir des ressources financières, les libéraux espagnols ont dû affronter d'autres "marchés émergents libéraux", tout particulièrement ceux de nouveaux pays indépendants comme les États latino-américains ou la Grèce. Et les exilés espagnols se sont heurtés à un obstacle majeur : le fait qu'ils n'étaient pas à la tête d'un gouvernement officiel et qu'ils avaient précisément dû s'expatrier en raison de leur implication dans la monarchie constitutionnelle, établie en Espagne durant le Triennat libéral et écrasée par une intervention française. Cependant, parmi les exilés politiques, se trouvaient quelques hommes d'affaire importants, des banquiers professionnels et d'anciens ministres des Finances qui ont mis au service du projet libéral leurs expériences et leurs contacts.

L'analyse de leurs activités nous amènera à considérer deux scènes géographiques, Londres et Paris, qui constituaient les principales destinations de l'élite de l'exil espagnol. Durant la première moitié du XIX ${ }^{e}$ siècle, ces deux villes ont forgé leur propre marché moderne de valeurs ainsi que d'autres nouveaux instruments financiers; elles se sont ainsi transformées en centres de l'activité bancaire mondiale. Les bourses des capitales britannique et française ont été cruciales pour le développement des liens qui ont étroitement 
uni le nord de l'Europe, la Méditerranée et le continent américain ${ }^{4}$. Au cours de ce processus, les exilés - de même que d'autres personnes en mouvement, comme les diplomates - ont contribué à redessiner l'économie internationale à travers l'utilisation de plusieurs instruments financiers, tout spécialement par le biais de l'émission et de la réémission de bons de dette publique sur les marchés mondiaux. Nombre d'entre eux figuraient également au centre de multiples connexions financières internationales qu'ils ont utilisées pour obtenir des financements. Un passage en revue des investisseurs avec lesquels les exilés politiques ont tenté d'entrer en contact constituera le second moment de notre analyse.

Enfin, nous complèterons notre parcours par une approche des changements de discours et des transferts institutionnels et politiques qui sont nés des relations tramées autour des activités financières des exilés, des gouvernements espagnols et des prêteurs avec lesquels les uns et les autres ont négocié des sorties face à une situation politique et économique critique, comme celle qu'a traversée l'Empire espagnol dès le début de son implosion en 1808 et jusqu'au-delà de la première guerre carliste (1833-1839) . La date finale de notre étude, l'année 1851, n'est qu'un point d'arrivée possible parmi d'autres : son choix est déterminé par la décision prise par le gouvernement de Juan Bravo Murillo de convertir la dette. Cette date ne doit pas être interprétée toutefois comme un point de rupture, mais plutôt comme une avancée importante dans le processus de réorganisation de la fiscalité et du crédit engagé par l'État libéral espagnol, qui s’est accéléré durant les années 1840 avec la promulgation de plusieurs lois.

\section{LES ACTIVITÉS FINANCIÈRES DES EXILÉS ESPAGNOLS}

Les exilés espagnols ont combattu la monarchie du roi Ferdinand VII non seulement à travers la lutte armée ou sur le champ de bataille de l'opinion publique internationale, deux aspects qui ont été jusqu'à présent les plus étudiés ${ }^{6}$, mais également par le biais de procédés financiers. Comme la majeure partie de leurs biens était confisquée en Espagne ${ }^{7}$, les exilés avaient

4. Au sujet de l'évolution des places financières, Youssef Cassis, Capitals of capital. A History of International Financial Centres, 1780-2005, Cambridge, CUP, 2005, offre une vaste description qui inclut une explication suggestive des avantages comparés de Paris qui ont permis à sa haute banque en formation de défier l'hégémonie de Londres après les guerres napoléoniennes. Le rôle joué par Paris durant les années 1820 et 1830, comme point de connexion entre l'espace régional de l'Europe du Sud et la sphère plus strictement anglo-saxonne, est d'un intérêt tout particulier pour notre travail.

5. Sur la crise des finances au cours de cette étape, $c f$. Juan Pan-Montojo, «La construcción del nuevo estado y la fiscalidad : España, 1808-1845», in Jaime O. Rodríguez (éd.), Las nuevas naciones: España y México, Madrid, Fundación Mapfre, 2008, p. 169-190.

6. Irene Castells, La utopia insurreccional del liberalismo. Torrijos y las conspiraciones liberales de la década ominosa, Barcelona, Crítica, 1989; Juan Luis Simal, Emigrados..., op. cit., chapitres 7 et 8.

7. La plupart des libéraux furent condamnés par l'application de la «loi seconde, titre second de la septième partida", des Partidas médiévales d'Alphonse X, qui fixait la peine de mort et la 
besoin d'obtenir de toute urgence des ressources monétaires : il s'agissait ainsi d'assurer leur propre subsistance mais aussi de renverser leur situation, de faire tomber la monarchie absolue espagnole et de pouvoir rentrer chez eux, même si, bien entendu, tous les exilés qui ont mobilisé des capitaux ne se sont pas lancés dans l'activisme politique. Ils pouvaient obtenir cet argent grâce à quatre ressources principales : le fruit de leur travail, les dons réalisés par des sympathisants de leur cause, les subventions concédées par les gouvernements des pays qui ont accueilli des réfugiés et enfin le maniement d'instruments financiers. Dans cet article, nous nous concentrerons sur ce dernier aspect - négligé par les historiens qui ont analysé la période -, sans pour autant écarter les trois premiers.

Il semble que les relations nouées entre politique et finance durant l'exil soient placées sous le sceau de l'ambiguïté. Certains exilés espagnols ont manifestement employé des instruments financiers pour s'enrichir personnellement, à l'exemple du plus heureux d'entre eux, Alejandro Aguado, qui devint l'un des plus grands banquiers français. Celui-ci n'était toutefois pas à proprement parler un libéral, puisqu'il avait gagné l'exil en France comme joséphin. Son cas nous conduit plutôt à considérer l'absence de liens entre positions politiques personnelles et monde de la finance, ainsi que l'importance de l'adaptation aux circonstances. Néanmoins, il met également en lumière la polyvalence de réseaux sociaux à la fois familiaux et politiques. Aguado, qui avait obtenu la nationalité française, était devenu l'agent financier de Ferdinand VII en France. C'est lui qui avait négocié la majeure partie de la dette de la monarchie espagnole - précisément lorsqu'un groupe d'anciens joséphins avait fini par occuper des positions de pouvoir au sein de l'État fernandin. Il avait alors pu s'enrichir par des moyens douteux ${ }^{8}$.

De nombreux autres exilés ont cependant réussi à faire des affaires et à réaliser des investissements privés tout en finançant des projets idéologiques qui impliquaient des changements politiques et économiques révolutionnaires. Les finances sont devenues un moyen pour la réalisation de leurs objectifs politiques, tout en représentant un instrument pour survivre dans l'exil ou encore, pour quelques-uns parmi les plus habiles, un moyen d'enrichissement personnel. Parmi les exilés espagnols, il y avait des spécialistes des finances comme Juan Álvarez Mendizábal, les frères Bertrán de Lis ou Lorenzo Calvo y Mateo, qui ont mis leur expérience et leurs contacts au service du projet libéral. Nombre d'entre eux étaient également au centre de réseaux financiers internationaux qu'ils ont mobilisés afin de lever des fonds. Mendizábal, par exemple, a joué un rôle central dans le financement des

confiscation des biens pour les délits de trahison; on consultera par exemple le procès de B. Gallardo décrit dans Alejandro Pérez Vidal, «La condena a muerte de Bartolomé José Gallardo en 1815», in Marieta Cantos Casenave et Alberto Ramos Santana (eds), La represión absolutista y el exilio, Cadix, Universidad de Cádiz, 2015, p. 151-166. 2009.

8. Jean-Philippe Luis, L'ivresse de la fortune. A. M. Aguado, un génie des affaires, Paris, Payot, 
conspirations libérales espagnoles, mais ses services ont été également vitaux pour que les libéraux portugais en exil puissent obtenir des fonds pour payer leurs activités militaires contre la monarchie portugaise de Don Miguel$^{9}$. En plus de jouer un rôle actif sur les marchés financiers, certains exilés importants comme Álvaro Flórez Estrada ou José Canga Argüelles ont consacré beaucoup de temps à l'étude théorique de l'économie et ont produit des travaux sur l'économie politique, les finances et la dette publique ${ }^{10}$.

Les activités des exilés sur les marchés financiers internationaux ont eu un caractère aussi bien formel qu'informel. Tout d'abord, ils se sont employés à propager des rumeurs sur le manque de fiabilité des bons ou sur les objectifs assignés à certaines opérations concrètes des représentants de la monarchie espagnole, afin de faire chuter leurs cours dans les bourses de Londres et de Paris ${ }^{11}$. Par ailleurs, ils ont demandé aux investisseurs étrangers de s'engager politiquement aux côtés du libéralisme et de ne pas acheter les bons d'un gouvernement tyrannique. Sur ces terrains comme sur d'autres, l'on a vu se mêler des comportements opportunistes du point de vue politique à des actions guidées par des intérêts économiques : il s'agissait parfois de faire chuter ou d'augmenter le prix des titres pour léser un rival ou privilégier un allié et, dans d'autres cas, pour obtenir un profit personnel ${ }^{12}$. Outre ces pratiques, qui étaient peu différentes de celles des autres spéculateurs, les exilés politiques ont su, chaque fois que cela était nécessaire, empêcher aussi bien individuellement que collectivement l'admission de la dette de la monarchie tant que celle émise par le régime constitutionnel du Triennat n'était pas préalablement reconnue sur le plan international; lors de la réinstauration du pouvoir absolu, Ferdinand VII avait en effet rejetée celle-ci et il avait refusé par la suite d'envisager de rembourser les créanciers étrangers et nationaux. Il réitérait de cette manière sa décision de 1814 de suspendre le paiement

9. Juan Pan-Montojo, "Juan Álvarez y Mendizábal. (1790-1853) : el burgués revolucionario", in Manuel Pérez Ledesma et Isabel Burdiel (eds), Liberales, Agitadores y Conspiradores: Biografias Heterodoxas Del Siglo XIX, Madrid, Espasa Calpe, 2000, p. 155-182.

10. Le Curso de economía politica de Flórez Estrada a été publié pour la première fois à Londres en 1828 et a connu un succès rapide : il a été réédité à Paris en 1831 et en 1833 (il a été alors traduit en français pour cette édition) et à Madrid en 1835. Il allait connaître par la suite plusieurs rééditions à de nombreuses occasions, et l'on doit y ajouter à cette liste une édition certainement pirate qui a vu le jour à Caracas en 1840; on trouve davantage d'informations sur cette œuvre dans l'édition critique qu'en a réalisée Salvador Almenar, Curso de economía política, Madrid, Instituto de Estudios Fiscales, 1980. Quant aux cinq volumes du Diccionario de Hacienda de Canga Argüelles, ils sont parus à Londres entre 1826 et 1827. En 1825, ce dernier avait également fait paraitre à Londres ses Elementos de la ciencia de la Hacienda. Ces deux œuvres furent rééditées en Espagne à partir de 1833.

11. C'était là l'avertissement que l'embassadeur espagnol à Paris faisait au gouvernement français à la fin de l'année 1829, Archives du Ministère des Affaires étrangères (AMAEF), Paris, Mémoires et Documents, Espagne, 390, ff. 76-77, Ofalia à Polignac, Paris, 23 novembre 1829.

12. La gestion intéressée de l'information est l'une des clés de la spéculation "active" sur les marchés de valeurs. Le seul trait distinctif de la période de la Restauration est peut-être qu'il s'agissait de marchés relativement réduits et émergents, dans lesquels le nombre d'acteurs encore limité permettait d'utiliser des moyens de diffusion d'information plus «artisanaux» et personnalisés. L'on trouvera une vision classique de l'action des insiders sur les marchés dans Charles Kindleberger, Manias, Panics, and Crashes. A History of Financial Crises, New York, Basic Books, 1978. 
des intérêts de la dette. Toutefois, en 1823, la mesure allait encore plus loin puisqu'elle impliquait sa non-reconnaissance et donc l'élimination de ces titres de tout tableau de dettes. De même qu'entre 1814 et 1820 , la décision prise par le gouvernement de Ferdinand VII en 1823 a provoqué la fermeture des marchés formels de prêt en Europe, et l'a obligé à recourir à d'autres sources de financement plus coûteuses ${ }^{13}$.

Outre ces activités financières assumées par les exilés politiques sur les marchés de valeurs, les gouvernements provisoires et les juntes en exil ont émis leurs propres bons afin de financer leurs activités politiques et insurrectionnelles, et ont demandé aux investisseurs étrangers de les acquérir. Les arguments qu'ils ont employés pour capter ces investissements tournaient autour de la dette des Cortès ainsi qu'autour des opportunités que l'ouverture de l'économie espagnole apporterait aux investisseurs internationaux. L'aspect financier de leur lutte contre la monarchie de Ferdinand VII les a obligés à créer des instruments financiers particuliers qui alliaient intérêts économiques et politiques, tels les bons émis par les exilés. Nous avons pour l'heure identifié au moins trois de ces émissions de bons, liées aux différentes factions entre lesquelles les exilés espagnols se sont répartis durant leur exil : les deux premières juste après la révolution de Juillet, le 26 septembre et le 4 octobre 1830, et la troisième le 4 juillet 1831 . Mendizábal a en outre financé quelques-unes des expéditions militaires qui ont traversé les Pyrénées en octobre 1830 - principalement celles placées sous la conduite de Francisco Espoz y Mina - en renégociant les titres du Triennat qu'il possédait avec le banquier français A. Ardoin. Ce dernier avait en effet assisté le gouvernement constitutionnel en 1822 lors d'une émission d'obligations ${ }^{14}$. Au cours de ces opérations qui se déroulaient à Paris, Mendizábal a défendu la formation d'une junte composée par quelques-uns des dirigeants libéraux en exil en France. Cette junte devait adopter la forme d'un gouvernement en exil pour offrir des garanties plus solides aux acquéreurs de dette et permettre d'obtenir de meilleures conditions pour le prêt ${ }^{15}$.

La première émission de bons (26 septembre 1830) connue sous le nom d' «emprunt national espagnol ", était le fruit d'un accord entre Vicente Bertrán de Lis, représentant du "Gouvernement espagnol provisoire», le banquier parisien Matthieu Touanne et le capitaliste Jean Bernard Hippolyte Molineu. Les agents français s'engageaient à prêter leur assistance afin que

13. On trouvera un panorama général de la dette espagnole au XIXe siècle dans Javier Moreno Lázaro, "La deuda externa y colonial. Contratos y mercados (1808-1920) ", in Carlos Álvarez-Nogales y Francisco Comín Comín (eds), Historia de la deuda pública en España (siglos XVI-XXI), Madrid, Instituto de Estudios Fiscales, 2015, p. 251-285.

14. Peter Janke, Mendizábal y la instauración de la monarquía constitucional en España (17901853), Madrid, Siglo XXI, 1974, p. 43-44.

15. Peter Janke appuie son affirmation sur les Memorias del general Don Francisco Espoz y Mina, Imprenta y estereotipia de M. Ribadeneyra, 1851, volume IV, p. 86-87, bien que Mina nie dans ce texte son caractère de direction politique et la réduise à une simple Junte des moyens et des ressources (Junta de medios y recursos). 
soit obtenu un prêt de 110 millions de francs sur les marchés financiers de Paris, Londres et Amsterdam en échange d'une commission. Le destin assigné à cet argent ne faisait pas de doute, puisque l'article 8 de l'accord stipulait qu'une partie des fonds serait versée en nature, sous la forme de "vivres",

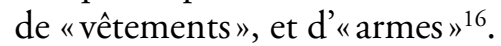

La seconde émission, en octobre 1830, a été réalisée à l'initiative de Lorenzo Calvo y Mateo, qui venait de regagner l'Europe depuis l'Asie où il s'était enrichi grâce au commerce de l'opium ${ }^{17}$. Elle consistait en un emprunt à court terme pour une valeur de 32400000 francs, en échange d'obligations émises à $35 \%$ de leur valeur nominale, ce qui représentait un taux d'intérêt élevé. Cet emprunt ne semblait pas jouir de l'appui des secteurs les plus importants de l'émigration espagnole, même si figuraient dans son comité de vigilance des exilés comme José Baiges et Juan Hernández, un ancien colonel. Calvo y Mateo aurait en outre envoyé de l'argent à Francisco Valdés, rival d'Espoz y Mina. L'emprunt n'a pas été souscrit dans sa totalité, principalement en raison de la date tardive à laquelle il avait été annoncé à la presse, les 12 et 31 octobre 1830, alors que les expéditions des Pyrénées avaient déjà échoué. De la sorte, les titres ont été rejetés et Calvo a dû se déclarer en banqueroute pour un montant de 2 millions de francs, avant de quitter la France pour se réfugier en Belgique ${ }^{18}$.

La troisième émission de bons (4 juillet 1831) a été réalisée par la «junte provinciale directrice de la Catalogne», présidée par le général Francisco Milans del Bosch et qui était liée au prêt des Cortès. Tout d'abord, la Junte a déclaré nulle et non avenue la conversion des titres de la dette des Cortès récemment décrétée par le gouvernement de Ferdinand VII (un gouvernement "tyrannique» et "absolutiste», selon leurs propres mots). En second lieu, l'on a annoncé l'ouverture à Paris et à Londres d'une nouvelle souscription adressée aux possesseurs de bons de crédit des Cortès et qui serait administrée par des comités «composés d'amis de la cause constitutionnelle d'Espagne». Les nouveaux titres seraient remboursés « dès qu'il existera[it] un gouvernement constitutionnel en Espagne, ou dès qu'une autorité constituée dans l'intérieur du pays aura[it] proclamé les droits du peuple» (article 12). Cette initiative a été communiquée au ministre français des Affaires étrangères, Sebastiani, par une lettre signée à Paris (le 17 août 1831) par les dirigeants autoproclamés de «l'Espagne libre», autrement dit du "gouvernement

16. AMAEF, Mémoires et Documents, Espagne 365, ff. 11-16.

17. Ander Permanyer Ugartemendia, "La participación española en la economía del opio en Asia oriental tras el fin del galeón ", thèse de doctorat d'histoire sous la direction de Dolors Folch Fornesa et de Josep Maria Delgado Ribas, Universitat Pompeu Fabra, 2013.

18. Robert Marrast, José de Espronceda et son temps. Littérature, société, politique au temps du romantisme, Paris, Éditions Klincksieck, 1974, p. 160, note 70; Peter Janke, Mendizábal..., op. cit., p. 48. 
constitutionnel ", c'est-à-dire par Manuel Bertrán de Lis, Antonio Baiges y Andrés Borrego ${ }^{19}$.

Ces émissions de bons ont-elles eu du succès? Quand et de quelle manière les prêts furent-ils remboursés? Quelles ont été les conséquences de ces engagements financiers pour la politique espagnole au cours des années qui ont suivi $?^{20}$ On trouve des preuves qui attestent que les exilés libéraux espagnols ont rencontré un certain succès dans leurs efforts pour lever des fonds grâce à ces procédés, même si le détail reste encore à déterminer. Bien évidemment, au cours des mois postérieurs aux émissions, les exilés ont lancé plusieurs tentatives d'invasion du territoire espagnol par les Pyrénées en utilisant le Sud de la France comme plateforme. Dans les rapports diplomatiques et policiers que nous avons analysés jusqu’à présent, de nombreuses références à plusieurs investisseurs français apparaissent, mais l'on peut encore s'interroger sur leur identité exacte ${ }^{21}$.

Les recherches qui restent encore à mener sur les exilés libéraux et sur leurs activités financières exigent également d'entrer dans l'histoire, elle, bien mieux connue, des prêts contractés par la monarchie espagnole au cours de la période 1823-183322. Comme nous avons pu le signaler, la décision de restaurer l'absolutisme en Espagne en 1823 et de ne pas reconnaittre la dette émise par les Cortès a fermé à la monarchie de Ferdinand VII les portes des bourses européennes. Cette décision l'a aussi obligé à négocier avec des banques et des intermédiaires financiers l'obtention de fonds, indispensables pour des finances publiques en crise. Nous en savons assez sur ces agents officiels et officieux de Ferdinand VII en Angleterre et en France : la majeure partie d'entre eux était liée aux afrancesados, dont Aguado était sans doute la figure la plus remarquable. En revanche, l'on a accordé peu d'intérêt au fait de relier leur activité avec celle des exilés, par-delà l'esquisse, dans ce contexte, des origines de groupes de financiers et politiciens rivaux, assortis d'interlocuteurs étrangers en général différents, qui s'affronteraient plus

19. AMAEF, Affaires Diverses Politiques, Espagne, 19.

20. En 1830, un franc français équivalait à peu près à quatre réaux de vellón, la principale unité monétaire du moment. Ainsi, pour donner un exemple, les 110 millions de francs de la première émission mentionnée équivalaient à 440 millions de réaux. Pour se faire une idée du volume réel de ces emprunts, il est utile de les comparer au budget de l'État espagnol en 1830 qui atteignait la somme de 593 millions de réaux, l'armée recevait 253 millions et la marine 41. Cette conversion a été réalisée à l'aide de historicalstatistics.org, selon les données présentées par Francisco Comín et Daniel Díaz, "Sector público y administrativo y estado del bienestar», in Albert Carreras et Xavier Tafunell (eds), Estadisticas históricas de España. Siglos XIX-XX, Bilbao, Fundación BBVA, 2005, t. 2, p. 910.

21. Cf. les documents insérés dans Manuel Núñez de Arenas, «Españoles fuera de España. La expedición de Vera en 1830 (según documentos inéditos de Policía) ", Boletín de la Real Academia de la Historia, 90, 1927, p. 610-666.

22. Sur l'histoire de la dette et de la fiscalité, outre l'œuvre classique de Josep Fontana, Hacienda y Estado, 1823-1833, Madrid, Instituto de Estudios Fiscales, 2001, on peut consulter Jean-Philippe Luis, L'utopie réactionnaire : épuration et modernisation de l'état dans l'Espagne de la fin de l'Ancien Régime (1823-1834), Madrid, Casa de Velázquez, 2002; et Miguel Ángel López Morell, La Casa Rothschild en España, Madrid, Marcial Pons, 2005. 
ouvertement après le triomphe du libéralisme ${ }^{23}$. Suivre l'évolution conjointe des stratégies des uns et des autres pour obtenir des ressources, qu'ils agissent comme des agents privés ou pour défendre leur propre cause, ainsi que pour priver leurs adversaires de leurs soutiens financiers, serait d'un grand intérêt : nous découvririons certainement les nombreuses possibilités qui se sont ouvertes pour des joueurs qui négociaient avec les deux parties, en parvenant à nouer une forme de complicité avec chacune d'entre elles.

\section{LES INVESTISSEURS ÉTRANGERS}

L'autre versant de toute entreprise financière est l'investissement. Les investisseurs qui ont acheté les bons espagnols étaient pour la plupart des «capitalistes» étrangers, essentiellement français et britanniques. Dans l'historiographie qui s'est penchée sur l'exil espagnol, on trouve des références à ces "capitalistes», même si leur identité et leur niveau d'engagement n'apparaissent pas encore de manière claire ${ }^{24}$. L'argent nécessaire pour acheter les équipements militaires et pour payer les hommes qui ont participé aux insurrections planifiées depuis la France et la Grande-Bretagne dans les décennies 1820 et 1830 a bien dû avoir une provenance. Il n'est cependant pas évident d'identifier de manière probante les investisseurs et leurs motivations. Notre recherche souhaite apporter des réponses à ces questions. D'un côté, il est logique de penser que ces individus devaient nécessairement être proches du libéralisme sur le plan politique, puisque ces prêts étaient des investissements à haut risque. En même temps, il ne semble pas que l'appui porté au libéralisme ait été un élément essentiel ou indispensable. Toutefois, nous posons comme hypothèse de travail que, le plus souvent, leurs intérêts économiques et politiques - comme cela se produisait pour les premiers exilés - pouvaient coïncider. Outre les investisseurs financiers, il existe de nombreux cas de dons aux exilés espagnols de la part de personnalités comme le marquis de La Fayette ou Robert Boyd, ainsi que par des membres des comités britanniques et français formés pour assister les réfugiés. Dans le cas britannique, ils incluaient des lords, des membres du Parlement, des militaires de haut rang ou encore des journalistes. Les «amis de Lord Holland», le cercle des exilés et amis britanniques du libéralisme ibérique qui a été actif au moins au

23. Jean-Philippe Luis, «La dette publique et la reconfiguration des relations entre les financiers et l'État dans la dernière décennie de l'Ancien Régime espagnol (1823-1834)", in Anne Dubet et Jean-Philippe Luis [dir.], Les Financiers et la construction de l'État. France, Espagne (XVII ${ }^{e}-X V I I I^{e}$ siècle), Rennes, Presses Universitaires de Rennes, 2011, p. 155-176, évoque un premier réseau espagnol formé autour de Ballesteros-Aguado qui se réorganiserait ensuite autour de Muńoz, puis s'intéresse à un second réseau autour de Toreno et de son banquier, les Rothschild londoniens, et enfin d'un troisième autour de Mendizábal.

24. L'on trouve des éléments chez Irene Castells, Utopía insurreccional..., op. cit., p. 136; ibidem, "Constitucionalismo, estrategia insurreccional e internacionalismo liberal en la lucha contra el Antiguo Régimen español (1823-1831)", Revista de História das Ideias, volume 10, 1988, p. 485-506. 
cours de la seconde et de la troisième décennie du XIX ${ }^{\mathrm{e}}$ siècle, ont fourni aux exilés espagnols l'accès à leurs propres fonds et à ceux des autres selon tous les indices que nous avons pu rassembler ${ }^{25}$.

Les investisseurs étrangers étaient impliqués dans l'instauration d'un État libéral en Espagne pour différentes raisons. Tout d'abord, ils voulaient obtenir le paiement des bons du gouvernement constitutionnel espagnol qu'ils détenaient. Cette dette s'est transformée en un élément central des activités financières menées par les exilés espagnols dans les années 1820-1830, car son rejet a laissé des centaines d'investisseurs internationaux dans une situation précaire. Dans ces circonstances, ces détenteurs d'une dette impayée pouvaient fournir une manne financière dont les exilés avaient besoin afin de mettre en marche leurs plans insurrectionnels. En juillet 1825, par exemple, la police française affirmait que :

"Les négociants intéressés dans les emprunts du gouvernement constitutionnel d'Espagne, ayant perdu tout espoir de recouvrer leurs capitaux, avaient formé le projet d'une expédition contre ce royaume. Des émigrés espagnols, italiens et français qui se trouvent en Angleterre, devaient en former le noyau, et être soutenus par neuf mille recrues anglaises : ces négociants avaient promis de fournir les munitions, les armes et l'argent nécessaires ${ }^{26}$.

Cet intérêt immédiat se combinait en second lieu avec la possibilité de transformer la monarchie néo-absolutiste espagnole en un État libéral, avec les opportunités économiques que ce changement pouvait offrir aux investisseurs internationaux. L'objectif de ces investisseurs était d'obtenir l'accès au marché espagnol grâce à l'ouverture d'opportunités commerciales inédites dans une économie qui, à cette époque-là, se trouvait sous le contrôle d'un nombre restreint de commerçants disposant de privilèges, et qui était encore marquée par l'existence de corporations et de monopoles. Ils espéraient que les libéraux ouvriraient l'économie et introduiraient le libre commerce. Toutefois, la monarchie de Ferdinand VII adoptait en même temps plusieurs mesures afin d'affaiblir l'ordre des corporations, de renforcer les institutions qui dépendaient du pouvoir central, et d'encourager la croissance économique au moyen de l'importation de politiques et d'institutions des "autres nations qui nous devancent en richesse», ce qui incluait l'adoption d'importantes réformes financières ${ }^{27}$. Par conséquent, les bons des Cortès du

25. Manuel Moreno Alonso, La forja del liberalismo en España. Los amigos españoles de Lord Holland, Madrid, Congreso de los Diputados, 1997.

26. Extrait d'un rapport adressé le 18 juillet 1825 au Ministre de Naples à Londres; AMAEF, Mémoires et Documents, Espagne, 385, ff. 187-189.

27. La phrase entre guillemets et l'idée du besoin de procéder à ce changement sont contenues dans le Mémoire qu'a présenté en 1829 Jodé López Juana Pinilla, qui occupait alors un poste élevé du ministère de l'Économie - celui de directeur général des Revenus - avec l'appui de son neveu et futur ministre des Finances dans les années 1840, Ramón de Santillán (Josep Fontana Lázaro, «José López 
Triennat libéral ont dû entrer en concurrence sur les marchés européens avec les nouveaux bons émis par la monarchie absolutiste à nouveau restaurée ${ }^{28}$.

En troisième lieu, de nombreux investisseurs voulaient également assurer leurs intérêts commerciaux dans les jeunes républiques hispano-américaines qui, sans la reconnaissance officielle du gouverment espagnol, rencontraient d'importantes difficultés pour établir des relations diplomatiques avec d'autres États européens. Cette situation d'incertitude affectait dans l'économie atlantique de nombreuses affaires dirigées par des commerçants européens. Après l'échec qu'avait connu le projet d'expédition de 1825 et que nous avons déjà mentionné (les autorités françaises croyaient que son annulation était due à l'«indiscrétion de ce général [Milans], qui a[vait] donné l'éveil au Gouvernement espagnol»), un rapport de la police affirmait que les "mêmes négociants n'[avaient] pas abandonné leur projet", et qu'ils avaient décidé d'appuyer une nouvelle expédition :

«Ils ont mis dans leurs intérêts les principaux négociants de l'Amérique espagnole, en leur faisant croire que le clergé d'Espagne faisait des efforts pour organiser trente mille hommes destinés à remettre l'Amérique sous le joug et c'est concert qu'ils ont résolu de tenter une expédition dans le double but de forcer le roi d'Espagne à reconnaitre les emprunts de Cortès et d'empêcher qu'il ne puisse tenter rien de sérieux contre les colonies insurgées, afin de leur donner le temps d'affermir leur indépendance, et s'armer sur terre et sur mer $»^{29}$.

L'appui apporté à la cause des nouvelles nations hispano-américaines a toutefois eu des conséquences pour les exilés espagnols : pour placer leurs bons sur le marché de la dette publique internationale, ils ont dû entrer en rivalité avec la dette émise par les républiques hispano-américaines, ainsi qu'avec d'autres marchés émergents libéraux comme ceux du Brésil et de la Grèce. Le principal problème que devaient affronter les républiques hispano-américaines provenait du fait que leurs agents diplomatiques étaient des représentants d'États dont l'existence n'avait pas encore été reconnue par la plupart des gouvernements européens : seule la Grande-Bretagne l'avait fait à la fin de l'année 1824. Les exilés espagnols affrontaient un défi similaire : il s'agissait de convaincre les investisseurs de financer un gouvernement non officiel en exil. Les États latino-américains ont obtenu un succès assez important dans l'obtention de ces fonds, même si la spéculation causée par les attentes démesurées éveillées par les immenses et presque fantasmatiques richesses américaines a joué un rôle important dans ce succès initial.

Juana Pinilla», in Enrique Fuentes Quintana (ed.), Economía y economistas españoles. 4. La economía clásica, Barcelone, Galaxia Gutenberg/Círculo de Lectores, 2000, p. 710).

28. Jean-Philippe Luis, L'utopie réactionnaire..., op. cit.

29. Extrait d'un rapport adressé le 18 juillet 1825 au ministre de Naples à Londres, AMAEF, Mémoires et Documents, Espagne, 385, ff. 187-189. 
Peu après, en 1825, la situation a débouché sur une grande crise de la dette lorsque la bulle spéculative a explosé sur le marché de Londres ${ }^{30}$.

Dans le même temps, les exilés espagnols ont collaboré avec les agents diplomatiques des pays latino-américains basés en Europe, pour publier des livres et des organes de presse à caractère de propagande, ou encore pour lancer des projets éducatifs et insurrectionnels. L'émissaire Vicente Rocafuerte, de nationalité mexicaine même s'il était né à Guayaquil (Équateur), a participé au financement de journaux et de livres publiés par des exilés libéraux espagnols, nombre d'entre eux étant destinés à être introduits et diffusés en Amérique Latine. La possibilité que les projets insurrectionnels espagnols puissent recevoir des fonds de l'Amérique latine s'est peu à peu imposée comme un fruit de ces contacts. Les agents des républiques hispanoaméricaines, parmi eux Rocafuerte, ont offert des ressources financières aux libéraux espagnols dans l'espoir qu'une fois que les exilés auraient atteint le pouvoir, ils normaliseraient les relations avec les nouvelles nations, menacées par l'absence de reconnaissance internationale mais également par les plans de reconquête qui n’ont jamais été abandonnés par Ferdinand VII. Toutefois, il semble que cette possibilité ne se soit jamais concrétisée par un financement significatif ${ }^{31}$. Le 25 mars 1828, Rocafuerte a signé avec des délégués de la Junte de Londres un traité par lequel les Espagnols s'engageaient à accepter l'indépendance du Mexique. Le gouvernement mexicain garantissait pour sa part qu'il concéderait à ces derniers un emprunt de cinq millions de pesos lorsqu'ils mettraient à exécution leurs projets insurrectionnels. Les difficultés politiques intérieures du Mexique à ce moment-là ont empêché la ratification du traité, mais les contacts se sont poursuivis en Angleterre. Gorostiza, le nouveau représentant mexicain, a remis peu après à Torrijos 100000 réaux "pour les bons et loyaux services qu'il avait rendus à la République du Mexique» ${ }^{32}$.

La circulation des bons émis par les exilés espagnols met enfin en évidence le cas des petits investisseurs qui ont acheté de la dette espagnole sur le marché secondaire. Ils disposaient de moins de ressources et d'influence, de sorte qu'ils sont restés pour la plupart sans la moindre protection face aux impayés. Toutefois, durant les décennies 1820 et 1830, ils ont lancé toute une série d'actions

30. Frank Griffith Dawson, The First Latin America Debt Crisis. The City of London and the 1822-25 Loan Bubble, New Haven et Londres, Yale University Press, 1990; Rebecca Cole Heinowitz, "The Spanish American Bubble and Britain's Crisis of Informal Empire, 1822-1826", in Joselyn Almeida (éd.), Romanticism and the Anglo-Hispanic Imaginary, Amsterdam-New York, Rodopi, 2010.

31. Cf. J. E. Rodríguez O., The Emergence of Spanish America. Vicente Rocafuerte and Spanish Americanism, 1808-1832, Berkeley-Los Angeles, University of California Press, 1975; Eugenia Roldán Vera, The British Book Trade and Spanish American Independence: Education and Knowledge Transmission in Transcontinental Perspective, Aldershot, Ashgate, 2003; María T. Berruezo, La lucha de Hispanoamérica por su independencia en Inglaterra, 1800-1830, Madrid, Ediciones de Cultura Hispánica, 1989; Juan Luis Simal, Emigrados..., op. cit., p. 333-339.

32. Irene Castells, Utopía insurreccional..., op. cit., p. 135-137; citation du rapport présenté par l'indicateur José Baiges aux autorités espagnoles, dans Archivo Histórico Nacional, Madrid, Estado, 3075, 31 mai 1830 . 
pour récupérer leur argent en recourant également à la formation d'associations et de comités, à la publication de feuillets et d'articles dans la presse, et à l'envoi de pétitions aux autorités. En France, au cours des années 1830, ils ont atteint la famille royale par le biais de pétitions qu'ils ont fait parvenir à la reine et au roi pour qu'ils intercèdent auprès du gouvernement et des chambres législatives $^{33}$. On retrouve ainsi un cas similaire à celui des possesseurs britanniques de bons mexicains, étudié par Michael P. Costeloe ${ }^{34}$.

\section{Discours et TRANSFERTS : INNOVATIONS CULTURELles ET INSTITUTIONNELLES}

Les exilés politiques libéraux ont associé à leurs activités financières des opérations discursives novatrices, intimement liées au transfert et à l'adaptation d'institutions financières étrangères ainsi qu'à la création de formules propres dans ce domaine en Espagne. Les exilés libéraux et leurs pourvoyeurs de fonds étrangers ont promu un changement dans le cadre général de l'économie espagnole par le biais de la revendication de réformes modernes. Ils aspiraient à établir un ordre économique plus ouvert, qui ait une influence positive sur leurs affaires privées et qui affermisse le nouvel État libéral. Pour ce faire, ils se sont prévalus de discours de progrès, de modernisation et de liberté, ce qui a eu une incidence remarquable sur la construction de l'État et sur la reconfiguration de la nation en Espagne.

Nous nous proposons ainsi de reconsidérer l'impact de l'expérience financière dans l'exil sur l'élaboration de politiques économiques, financières et fiscales de l'État libéral en Espagne à partir de 1833, et même avant cette date, grâce au rôle joué par d'anciens afrancesados dans les appareils absolutistes tout au long des années 1820. Après la mort de Ferdinand VII, en 1833, les exilés libéraux sont rentrés en Espagne et nombre d'entre eux ont à nouveau occupé des postes au sein du gouvernement. Les réformes et projets institutionnels que les uns et les autres ont appuyés, en relation directe avec le financement extérieur, se distribuent en trois grands champs : le domaine strictement financier, celui du désamortissement et la fiscalité.

Avant la fin de la monarchie absolue, il y a eu un ensemble de changements institutionnels qui ont été directement liés aux finances et, plus spécifiquement, au besoin absolutiste d'obtenir des crédits sur les marchés européens, bien qu'il ne convienne pas non plus de les séparer de plans à plus long terme. Entre 1829 et 1831, le réformisme absolutiste a approuvé des mesures d'une grande importance politique qui ont affecté son activité commerciale et financière : le Code du Commerce (1829), la loi de procédure

33. Cf. les documents et pétitions, AMAEF, Affaires Diverses Politiques, 1814-1896, Espagne, 19.

34. Michael P. Costeloe, Bonds and Bondholders: British Investors and Mexico's Foreign Debt, 1824-1888, Westport, Praeger, 2003. 
commerciale (1830), l'établissement de la banque de San Fernando (1829) et l'ouverture d'une Bourse de valeurs à Madrid (1831). Ces lois ont toutes été liées à un personnage controversé de l'époque, le joséphin revenu d'exil Pedro Sainz de Andino, qui avait été coopté par le dirigeant du réformisme fernandin et ministre des Finances, Luis López Ballesteros. Sainz de Andino, juriste appartenant au réseau construit autour de Ballesteros, a été amené à rédiger de nombreux projets de loi à la demande de ce dernier, mais également d'autres individus - espagnols et étrangers - qui connaissaient sa position privilégiée dans l'organigramme du ministère ${ }^{35}$. Son passage par la France et ses liens avec les autres joséphins lui donnaient également d'autres atouts plus culturels : c'était un juriste très attentif aux nouveautés qui se produisaient en France. Dans sa bibliothèque, $42 \%$ des livres de droit étaient d'ailleurs en français $^{36}$. Toutes ces lois rédigées par Andino et approuvées avec le soutien du réformisme absolutiste supposaient des avancées d'une grande importance politique, comme l'ont bien sûr mesuré en retour les secteurs ultra-royalistes. Tout d'abord, le Code du Commerce de 1829 a codifié un droit spécial au sein du droit privé, bien avant que l'on ne puisse en faire de même avec le droit civil, et il soumettait les corporations de marchands et négociants à la régulation de l'État. En second lieu, le processus déjà enclenché d'uniformisation, d'étatisation et de nationalisation de la juridiction mercantile a été réaffirmé par la loi de procédure commerciale. De son côté, l'ouverture d'une bourse du commerce à Madrid permettait d'améliorer la position de la monarchie qui cherchait à obtenir du crédit intérieur. La fondation de la banque de San Fernando a été une tentative de créer une banque centrale comparable à celle de la France, qui était son modèle ${ }^{37}$. À l'évidence, tous ces progrès ainsi que d'autres réalisés par le réformisme absolutiste n'ont pas été une simple transposition d'institutions inspirées par la législation française, et encore moins des mesures imposées par les créanciers. Ils révèlent quel était l'axe du programme réformateur d'un groupe dont la tâche politique principale et dont la grande affaire collective ont consisté à lever des fonds pour la monarchie sur le marché français des capitaux, le seul auquel ils avaient vraiment accès grâce, en bonne partie, aux portes que leur avaient ouvertes les anciens afrancesados.

Le désamortissement des biens de l'Église ne peut pas être non plus séparé des problèmes financiers de la monarchie. Il a été lancé en pratique par le gouvernement du Comte de Toreno, ancien exilé, durant l'été 1835, même

35. Sur les références et la documentation qu'il a employées dans la rédaction des Codes du commerce, $c f$. Carlos Petit, «El Código de Comercio de Sainz de Andino (1829). Algunos antecedentes y bastantes críticas", Revista de derecho mercantil, 289, juillet-septembre 2013, p. 109-151.

36. Carlos Petit, «El legislador y la biblioteca. Los fondos de Andino en la Universidad de Sevilla», GLOSSAE. European Journal of Legal History, 10 (2013), p. 489-506, (disponible sur http:// www.glossae.eu).

37. Pedro Tedde, El Banco de San Fernando (1829-1856), Madrid, Alianza/Banco de España, 1999. 
si la première loi de nationalisation et de vente des terres de l'Église a été approuvée alors qu'était ministre de l'Économie un autre homme récemment revenu d'exil Juan Álvarez Mendizábal - qui était, comme nous l'avons mentionné, l'un des principaux banquiers espagnols durant l'exil - à l'automne de cette même année. Si «la libération des terres du joug de l'Église» faisait partie de nombreux projets de lois agraires à l'époque des Lumières, et si la construction d'un nouveau marché agraire a constitué un objectif du libéralisme depuis Cadix, «créer le maximum de produits pour amortir le plus possible le capital de la dette publique» était, en 1836, la priorité de l'opération de désamortissement selon Mendizábal. De même, en 1855, le but allait être d'obtenir "des revenus pour couvrir les obligations de l'État», selon les mots d'Antonio González, président de la commission qui étudiait aux Cortès le projet de loi de désamortissement axé sur les biens communaux ${ }^{38}$.

Bien évidemment, depuis les mesures extraordinaires adoptées par le ministre Godoy à partir de la fin du XVIII siècle, le désarmortissement était devenu la meilleure façon d'obtenir des moyens exceptionnels et de permettre à l'État de faire face à ses créanciers. C'est ce qui s'est produit dans le Madrid joséphin, à Cadix et durant le Triennat : la Banque de San Carlos a tenté de trouver à plusieurs reprises dans les biens nationaux une voie de compensation pour les dettes qu'avaient contractées auprès d'elle les finances royales $^{39}$. Mais un argument semblable a été également avancé lors de la seconde restauration de l'absolutisme, même si cela a été depuis un point de vue inverse : en 1825, les banquiers Rothschild ont demandé que l'on emploie le patrimoine de l'Église pour assurer un prêt de Ferdinand VII ${ }^{40}$. Les difficultés dont souffrait le crédit intérieur et extérieur de l'Espagne ont été par ailleurs la justification la plus directe et explicite de la forme adoptée par le désamortissement de Mendizábal en 1835-1836 et par celui de Madoz en $1855^{41}$. La réforme agraire libérale, par-delà ses objectifs à moyen terme, a donc été guidée dans ses contenus, ses formats et ses rythmes par les exigences dérivées de la gestion de la dette et par les pressions concrètes exercées par les créanciers aussi bien intérieurs qu'extérieurs. Les terres de

38. Se reporter à l'Exposición de motivos del Real decreto de 19 de febrero de 1836 et Diario de Sesiones de Cortès Constituyentes, 28.III.1855, p. 3309.

39. Pedro Tedde, El Banco de San Carlos, Madrid, Alianza/Banco de España, 1988, p. 375.

40. López Morell, La Casa Rothschild..., op. cit., p. 56.

41. Comme l'ont déjà expliqué les spécialistes du XIX siècle, le désarmortissement de Mendizábal a eu pour objectif principal d'amortir de la dette intérieure : les titres de cette dette ont été ceux qui ont pu être employés pour l'acquisition de biens immeubles. Mais cette opération (décrite avec des données actualisées par Rafael Vallejo Pousada, «Las vicisitudes de la deuda pública en un período turbulento : de Canga Argüelles a Mon, 1808-1850 ", in Carlos Álvarez-Nogales et Francisco Comín Comín (éds.), Historia de la deuda pública en España (siglos XVI-XXI), Madrid, Instituto de Estudios Fiscales, 2015, p. 159-186), a été liée à la dette extérieure, dans la mesure où la réduction du volume total de la dette devait augmenter le crédit de la monarchie. Une relation semblable entre désamortissement et crédit intérieur et extérieur fut établie par Madoz en 1855. $C f$. Juan PanMontojo, «Pascual Madoz e Ibáńez : perfil de un progresista isabelino», in Francisco Comín, Pablo Martín Aceńa et Rafael Vallejo (eds), La Hacienda por sus ministros. La etapa liberal de 1845 a 1899, Saragosse, Prensas Universitarias de Zaragoza, 2006, p. 171-208. 
mainmorte n'ont en outre pas été les seules ressources dont l'affectation a été conditionnée par le crédit. Les richesses existantes dans le sous-sol espagnol - dans le cas des intérêts des Rothschild, tout particulièrement le mercure, qui était indispensable pour l'obtention de l'argent - ont été pendant de longues années l'une des contreparties permettant à la monarchie d'avoir accès au crédit. L'histoire des concessions minières et, partant, de la législation minière n'a de sens que dans le contexte de l'obtention et de la renégociation de fonds sur les marchés internationaux ${ }^{42}$.

Le processus de transformation lié à l'endettement extérieur est enfin lié aux réformes des finances publiques. Au cours des décennies suivantes, le retour des exilés a suscité un flux continu de nouveautés aussi bien en ce qui concerne les impôts - qui, en 1845, ont été réformés en profondeur et adaptés aux nouveaux principes de l'État libéral - que pour les institutions de la dette et sa gestion, qui ont débouché sur les réformes entreprises par Bravo Murillo en 1851. La dette extérieure et les détenteurs étrangers n’ont évidemment pas été les seules forces de soutien au réformisme, mais ils ont joué un rôle déterminant et ont fourni des modèles à partir desquels les réformes des finances espagnoles ont pu émerger. Sur ce terrain, le secteur des taxes douanières a été, comme l'on pouvait s'y attendre, celui sur lequel la monarchie espagnole a reçu le plus de pression directe de la part des créanciers internationaux et de leurs gouvernements. Comín et Vallejo avancent qu'entre 1834 et 1843 le réformisme douanier a été guidé par l'«influence britannique» aussi bien sous le ministère présidé par Toreno que, plus tard, dans les cabinets progressistes de Mendizábal et Espartero ${ }^{43}$.

De manière plus générale, il est assez clair que, sur le plan politique, la dépendance financière a eu des conséquences décisives. Les relations des exilés avec les investisseurs étrangers et les possesseurs de bons ont tissé un ensemble de réseaux de grande importance pour la politique des premières décennies de l'État libéral espagnol. Les ambassades britannique et française à Madrid sont devenues des centres influents. Les libéraux progressistes - membres de l'un des deux partis autour desquels s'est organisé le libéralisme durant le règne d'Isabelle II, l'autre étant connu sous le nom de parti modéré - ont développé les réseaux qui avaient été créés durant leur exil anglais. Le Premier ministre et ministre des Finances Mendizábal a préservé ses contacts britanniques durant les années suivantes ${ }^{44}$. De la même manière, le général Espartero, régent d'Espagne entre 1840 et 1843, a entretenu une étroite relation avec les détenteurs de bons britanniques. De leur côté, les modérés ont en général développé des liens plus forts avec les investisseurs

42. Gérard Chastagneret, L'Espagne, puissance minière dans l'Europe du XIX'e siècle, Madrid, Casa Velázquez, 2000.

43. Francisco Comín Comín et Rafael Vallejo Pousada, Alejandro Mon y Menéndez (18011882). Pensamiento y reforma de la Hacienda, Madrid, Ministerio de Hacienda, 2002.

44. Juan Pan-Montojo, "Juan Álvarez y Mendizábal...», op. cit. 
français. Par le biais de Remisa, Casa Irujo ou Gaviria - qui appartenaient au cercle de Muñoz, le mari de la mère de la reine Isabelle II, dont l'influence a été si forte sur la vie politique et économique des années 1840 - les gouvernements modérés ont hérité de connexions établies autour de López Ballesteros à la fin de la période fernandine.

Comme l'illustre ce dernier cas, les réseaux n’ont pas été éphémères et n'ont pas souffert d'un progressif affaiblissement au cours des décennies 1830 et 1840 . L'instabilité du système politique espagnol a produit avec une certaine continuité de nouvelles vagues d'exil qui ont permis à leurs membres d'établir de nouveaux contacts. L'investissement étranger en Espagne a souvent employé les accès fournis par ces réseaux et, ce faisant, les a revigorés. En outre, ces relations n'ont pas seulement été utilisées à des fins privées : elles ont servi d'appui à des revendications politiques, elles ont permis le transfert d'idées et d'institutions, elles ont défendu des options politiques et économiques. La construction des institutions politiques dans l'exil a ainsi été intimement liée à la nécessité d'appuyer les émissions de dette. Il est vrai que ces institutions renvoyaient à leur tour à la tradition espagnole de constitution de juntes, mais elles impliquaient également une vision novatrice de l'espace international et du rôle des finances dans celui-ci. En second lieu, au cours du long conflit qui a opposé les partisans du protectionnisme et ceux du libre commerce en Espagne à partir des années 1840, les réseaux de l'exil ont nourri d'idées, de références et de propagande les porte-parole de ces deux pôles politico-économiques. En somme, les contacts et les réseaux ont subsisté, alors que par ailleurs l'État espagnol se transformait en une puissance de second ordre, subordonnée au Royaume-Uni et à la France. La fascination intellectuelle à l'égard des mondes rencontrés durant l'exil s'est prolongée, mais ceci n’implique cependant pas que la politique espagnole ait été le seul résultat des forces extérieures.

Les protecteurs financiers des libéraux espagnols - tout d'abord, lorsqu'ils se trouvaient en exil, durant la crise et, plus tard, lorsqu'ils étaient à la tête des institutions de l'État libéral - n'avaient pas à se sentir nécessairement proches d'eux en termes politiques. Parmi les "capitalistes", il y avait de grandes banques internationales comme Baring, Laffitte, Rothschild ou Guébhard, et également des possesseurs communs de bons et des négociants de niveau intermédiaire. Nombre d'entre eux ne voulaient obtenir que des bénéfices et du rendement pour leurs placements, ou s'intéressaient à la récupération de leur argent après une crise financière. La diversité des investisseurs britanniques et français liés à la dette espagnole, qui se différenciaient par leurs intérêts fiduciaires, commerciaux et économiques, mais aussi par leurs orientations et alliances politiques, dans leurs pays respectifs comme en Espagne, s'ajoutait à l'hétérogénéité qui séparait les politiques, fonctionnaires et groupes de pressions espagnols entre eux. Dans le jeu de ces réseaux transnationaux, variables dans le temps, des situations d'hégémonie 
et de dépendance n'ont vu le jour que dans des conjonctures politiques très particulières. De fait, la législation et la pratique des douanes, les décisions de désamortissement, les règlements et la structuration des mines, les progrès successifs qui ont façonné l'édifice juridique de l'État espagnol, seraient incompréhensibles si nous supposions que le crédit extérieur avait donné lieu à une relation de dépendance prolongée dans le temps, ou qu'il avait transmis des intérêts et des instructions homogènes à des groupes uniformes et passifs. L'histoire de la politique commerciale révèle au contraire l'ample marge de manœuvre des gouvernements et des parlements face aux pressions plurielles des créanciers et de la diplomatie de leurs pays respectifs. Mais cette histoire de la politique commerciale, et même celle des autres facettes de la politique espagnole du second tiers du XIXe siècle, mettent également en évidence que l'évolution institutionnelle et politique de l'Espagne resterait insaisissable si elle n'incluait pas les relations financières des exilés avec la monarchie, leurs interlocuteurs et associés, leurs relations cimentées par les liens économiques et leur influence sur la configuration de l'État libéral.

\section{EN GUISE DE CONCLUSION :}

PERSPECTIVES DE RECHERCHE

L'hypothèse de Karl Polanyi, exposée dans La Grande Transformation (première édition en 1944), sur le rôle décisif des centres financiers d'Europe occidentale dans le maintien de la période relativement longue de paix entre 1815 et 1914, a été un point de départ de nos hypothèses de recherche. Il s'agissait pour nous de savoir si ce qui semblait être de simples investissements financiers privés tirant profit des besoins croissants des États alors en construction, n'avaient pas eu, de fait, un sens politique plus vaste. Autrement dit, si les investisseurs et prêteurs n'avaient pas seulement joué un rôle de médiateurs dans des conflits internationaux potentiels, ce que Polanyi soutenait, mais également un rôle de soutien à des projets politiques, à des modèles de gouvernement et d'institution, en bref, s'ils n'avaient pas transféré leur savoir-faire capitaliste au-delà de leurs affaires personnelles, comme l'ont fait à partir du milieu du $\mathrm{XX}^{\mathrm{e}}$ siècle des institutions financières internationales comme le Fonds Monétaire International.

Dans l'historiographie espagnole, seul le secteur réduit des auteurs antilibéraux, autrement dit, ceux qui ont défendu le caractère non espagnol du libéralisme, a étudié l'influence des détenteurs de dette - les banquiers et les commerçants étrangers - dans la politique espagnole ${ }^{45}$. Mais, même parmi

45. La dépendance et la subordination des projets libéraux espagnols à des auteurs, des fonds et des appuis politiques britanniques et français a été soulignée sous le franquisme par ce que l'on appelle l'École de Navarre (Escuela de Navarra), dirigée par Federico Suárez Verdeguer, mais cette vision a encore certains partisans et continuateurs de nos jours. On se reportera, par exemple, au 
ces auteurs, aucun n'a tenté d'étudier leur influence sur les institutions et les discours. La plupart des historiens ont fondamentalement traité la dette extérieure comme un problème fiscal. Ils ont considéré les prêteurs privés comme des spéculateurs hostiles qui fomentaient la corruption des hommes politiques et des hommes d'affaires espagnols, et qui profitaient de leur position de pouvoir. Notre objectif est d'intégrer l'étude des intérêts, des discours politiques et du monde culturel sous-jacent dans lequel vivaient les prêteurs et les investisseurs, ainsi que les intermédiaires et les prestataires, afin de mieux saisir le processus de construction de l'État libéral espagnol et, de manière plus générale, le processus peu compris de diffusion et d'hybridation des idéologies, programmes politiques et institutions financières entre les centres et les périphéries du XIX ${ }^{\mathrm{e}}$ siècle.

Nous ne croyons pas que la construction de l'État libéral espagnol ait été déterminée par ceux qui ont sauvé, depuis Paris et Londres, leurs gouvernements de la banqueroute et ont donné à leurs soutiens politiques des ressources avec lesquelles triompher de leurs ennemis. Mais il n'est pas non plus possible de comprendre la trajectoire du libéralisme espagnol dans des termes exclusivement nationaux et politiques. La banqueroute fiscale de la monarchie espagnole au cours des guerres civiles qui ont accompagné la fin de l'Empire et de l'Ancien Régime n'a pas seulement été décisive à court terme; les ressources qui ont financé les projets du réformisme absolutiste et du libéralisme n'ont pas été un simple épisode secondaire. Revenir à l'analyse de la dette, des créanciers et débiteurs de la première moitié du XIX ${ }^{\mathrm{e}}$ siècle - une perspective de travail qui a constitué, voici quelques années, l'axe des recherches de pionniers comme Miguel Artola et Josep Fontana -, pour y inclure les exilés et leurs réseaux espagnols et transnationaux apparaît ainsi comme une piste féconde. Il devient ainsi possible d'analyser de manière plus satisfaisante la façon dont la relation de crédit a conditionné les alignements politiques et les relations internationales des élites libérales, et la manière dont elle a contribué à l'importation et à l'adaptation d'institutions étrangères ainsi qu'à la transformation et à la réorientation des institutions espagnoles.

Juan Pan-Montojo et Juan Luis Simal sont historiens à l'Universidad Autónoma de Madrid (Espagne)

Article traduit de l'espagnol par Philippe Rabaté

livre de Manuel Rodríguez Alonso, España y Gran Bretaña : diplomacia, guerra, revolución y comercio (1833-1839), Madrid, Actas, 1991. 